\title{
Anomalous Spin Dephasing in (110) GaAs Quantum Wells: Anisotropy and Intersubband Effects
}

\author{
S. Döhrmann, ${ }^{1}$ D. Hägele, ${ }^{1}$ J. Rudolph, ${ }^{1}$ D. Schuh, ${ }^{2}$ M. Bichler,${ }^{2}$ and M. Oestreich ${ }^{1}$ \\ ${ }^{1}$ Institut für Festkörperphysik, Universität Hannover, Appelstraße 2, D-30167 Hannover, Germany \\ ${ }^{2}$ Walter Schottky Institut, Technische Universität München, Am Coulombwall, D-85748 Garching, Germany
}

\begin{abstract}
A strong anisotropy of electron spin decoherence is observed in GaAs/(AlGa)As quantum wells grown on (110) oriented substrate. The spin lifetime of spins perpendicular to the growth direction is about one order of magnitude shorter compared to spins along (110). The spin lifetimes of both spin orientations decrease monotonically above a temperature of 80 and $120 \mathrm{~K}$, respectively. The decrease is very surprising for spins along (110) direction and cannot be explained by the usual Dyakonov Perel dephasing mechanism. A novel spin dephasing mechanism is put forward that is based on scattering of electrons between different quantum well subbands.
\end{abstract}

The electron spin in semiconductors has recently become a focus of intense research in the context of spinelectronics or spintronics. This new kind of electronics aims to utilize spin for devices with unprecedented properties [1, 2, 3]. A prime condition for the development of potential applications is the understanding of spin decoherence, i.e. the loss of spin memory, in semiconductor structures [4]. The main reason for spin decoherence at room temperature is the intrinsic spin splitting of the conduction band, which occurs in all binary semiconductors. The spin splitting, which acts as an effective magnetic field, depends on the electron's momentum and is the basis for the Dyakonov-Perel (DP) spin relaxation mechanism [5, 6]. Semiconductor heterostructures are in this context of particular interest since spin splitting in conduction and valence band can be controlled via dimensionality and orientation of crystal axes [7]. Ohno et al. observed very long electron spin decoherence times at room temperature in GaAs quantum wells (QWs) grown on (110) oriented substrates that exceeded the coherence times in usual (100) grown QWs by more than one order of magnitude [8, 9]. However, slow spin dephasing in (110) QWs had been demonstrated only for electron spins oriented along the crystal growth direction. The dynamics of in-plane spin was left unexplored.

Starting point for the theoretical description of the spin dynamics in (110) QWs is the Dresselhaus-Hamilton for binary semiconductors

$$
H_{\text {spin }}=\Gamma \sum_{i} \sigma_{i} k_{i}\left(k_{i+1}^{2}-k_{i+2}^{2}\right),
$$

where $i=x, y, z$ are the principal crystal axes with $i+3 \rightarrow i, \Gamma$ is the spin-orbit coefficient for the bulk semiconductor, and $\sigma_{i}$ are the Pauli spin matrices [10]. Comparing eq. (11) with the spin Hamilton for a free electron in a magnetic field $\left(H=\frac{1}{2} \sum_{i} \mu_{\mathrm{B}} \sigma_{i} B_{i}\right)$ one easily recognizes that random scattering of electrons leads to an effective $k$ dependent random magnetic field with components in $x, y$, and $z$ direction. This random magnetic field destroys the average spin orientation of an ensemble of electrons by rotating individual spins in different directions. The DP effect increases in bulk semiconductors with temperature due to occupation of higher $k$-states with larger spin splittings despite a motional narrowing effect at higher temperatures (spin lifetime $\tau_{s}$ is inversely proportional to momentum scattering time $\tau_{p}^{*}$ ). In (110) QWs, however, the spin splitting (effective magnetic field)

$$
H_{\mathrm{DP}}=-\Gamma \sigma_{z} k_{x}\left[\frac{1}{2}\left\langle k_{z}^{2}\right\rangle-\frac{1}{2}\left(k_{x}^{2}-2 k_{y}^{2}\right)\right],
$$

is suppressed in the plane of the QW via averaging of $H_{\text {spin }}$ along the confinement direction $z \|$ [110] (Ref. [10]). Here, $\left\langle k_{z}^{2}\right\rangle=\int\left|\nabla \psi_{z}\right|^{2} d z, \psi_{z}$ denotes the $z$ part of the electron wavefunction in the lowest subband, $x \|[1 \overline{1} 0]$, and $y \|$ [001]. An effective magnetic field exists only along the growth direction $z$. Electron spins oriented along $z$ are therefore not affected by the DP mechanism. In contrast, electrons with a spin component along $x$ or $y$ will randomly precess around the effective field, giving rise to spin dephasing anisotropy with dephasing rates $\gamma_{x}=\gamma_{y} \gg \gamma_{z}$.

In the first part of the paper we report on the observation of such a huge dephasing anisotropy with fast dephasing of electron spins oriented in the plane of a (110) QW and long dephasing times for spins along growth direction. The sample under investigation is a symmetrically modulation doped $\mathrm{GaAs} / \mathrm{Al}_{0.4} \mathrm{Ga}_{0.6} \mathrm{As}$ multiple quantum well (MQW) with 10 wells of $20 \mathrm{~nm}$ thickness and an electron density of $n=1.1 \cdot 10^{11} \mathrm{~cm}^{-2}$ per well. The sample is mounted in a magnet cryostat with variable temperature. The magnetic field lies in the plane of the MQW along the $y$ direction. Spin dephasing rates are determined by means of time and polarization resolved optical photoluminescence (PL) measurements. Spin oriented electrons are optically created in the sample along the growth direction by circularly polarized 100 fs pulses from a modelocked Ti:Sapphire laser with a repetition rate of $80 \mathrm{MHz}$. The excitation energy is at $1.675 \mathrm{eV}$, i.e. about $200 \mathrm{meV}$ above the PL energy. After excitation the carrier momentum distribution rapidly thermalizes by emission of phonons and scattering with other carriers. Holes rapidly lose their spin orientation due 


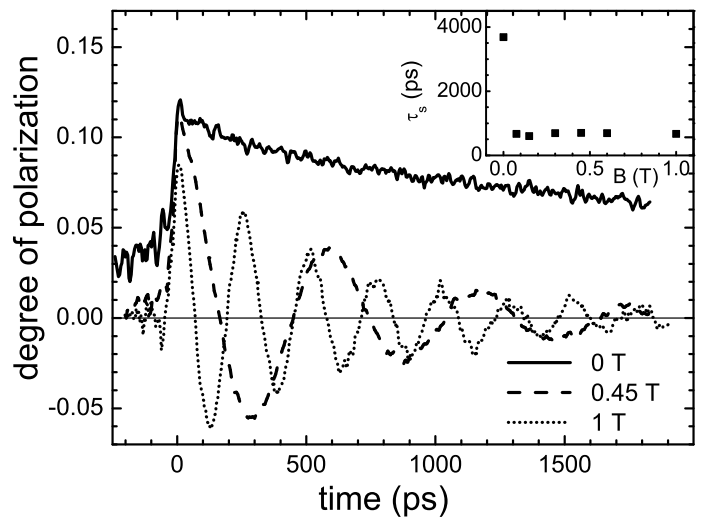

FIG. 1: Degree of polarization of the time resolved PL for magnetic fields of 0 (solid line), 0.45 (dashed line) and $1 \mathrm{~T}$ (dotted line) measured at a temperature of $200 \mathrm{~K}$. Inset: Dependence of measured spin lifetime $\tau_{s}$ on magnetic fields.

to strong valence band mixing and $k$ dependent spin splitting. The polarized luminescence is spectrally and temporally resolved by a synchroscan streakcamera with two-dimensional readout which provides a resolution of $1 \mathrm{~nm}$ and $20 \mathrm{ps}$, respectively. Polarization is selected by a liquid crystal retarder and a polarizer [11]. The optical selection rules yield a proportionality between the degree of circular PL polarization and the degree of spin orientation, with opposite sign for heavy hole (hh) and light hole (lh) transitions [6]. Photoluminescence lifetimes increase with temperature from $500 \mathrm{ps}$ at $6 \mathrm{~K}$ to $8 \mathrm{~ns}$ between $200 \mathrm{~K}$ and $300 \mathrm{~K}$, which is sufficiently long for a precise determination of spin relaxation times.

Figure 1 depicts the transient decay of circular polarization after excitation of about $2 \cdot 10^{10}$ carriers per $\mathrm{cm}^{2}$ at a sample temperature of $200 \mathrm{~K}$. For $B=0$, the average spin direction is always oriented along the $z$ direction where the DP mechanism does not apply. We find a long spin lifetime $\tau_{z}=\gamma_{z}^{-1}=3700 \mathrm{ps}$ as reported by Ohno [8]. For $B>0$ we observe spin precession around the field which leads according to the optical selection rules to a periodic change between $\sigma^{-}$and $\sigma^{+}$polarized luminescence in $z$ direction 12]. The magnetic field forces the spin after a quarter precession period in the plane of the QW, where the effective fluctuating magnetic field rapidly randomizes the spin orientation of the ensemble of electrons. We measure for the precessing spin an average dephasing rate $\left(\gamma_{z}+\gamma_{x}\right) / 2=1 / 670 \mathrm{ps}^{-1}$ which is by a factor of five faster than for spin in $z$-direction. The dephasing rate $\gamma_{x}=1 / 370 \mathrm{ps}^{-1}$ is even ten times larger than $\gamma_{z}$. Such a strong anisotropy in a semiconductor structure has never been reported before [13]. The rapid dephasing rates $\gamma_{x}$ and $\gamma_{y}$ have direct negative implications for devices where the stability of spins with arbitrary orientation is important, like in qubits for quantum computation. We measure the magnetic field dependence of the spin dephasing time to verify that the fast spin

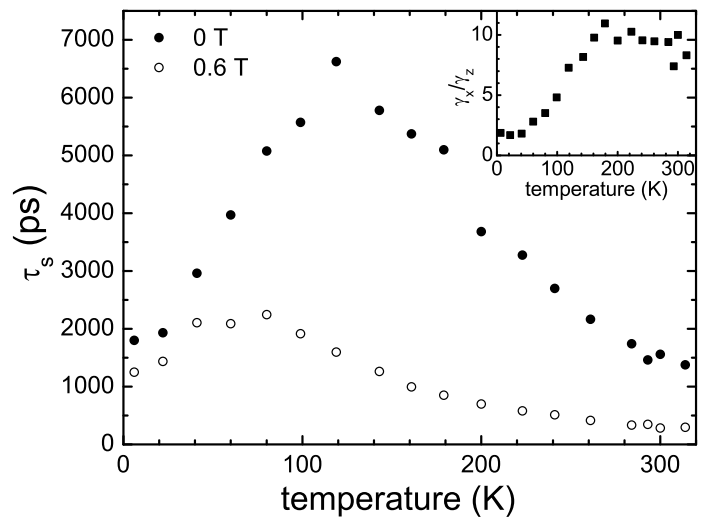

FIG. 2: Temperature dependence of spin lifetime $\tau_{s}$ for $B=0$ (closed circles) and $B=0.6 \mathrm{~T}$ (open circles). Corresponding temperature dependence of spin relaxation anisotropy $\gamma_{x} / \gamma_{z}$ (inset).

dephasing is not a new dephasing effect caused by the external magnetic field or inhomogeneous broadening of the precession frequency (inset of Fig. 1). The almost constant spin lifetime for $B>0$ clearly excludes a direct influence of the magnetic field on spin dephasing. We note that this spin dephasing for an electron precessing around an in-plane magnetic field is fully understood in the DP one-electron picture with an effective field in $z$ direction and does not require an additional "many-body inhomogeneous broadening effect" as claimed by Wu [14].

Next, we systematically investigate the spin lifetimes for temperatures between 6 and $314 \mathrm{~K}$ keeping the excitation power of the laser fixed. Figure 2 depicts the temperature dependence of the spin lifetimes at $B=0.6 \mathrm{~T}$ and $B=0$ for the full temperature range. Two features, the weak dephasing anisotropy at low temperatures $\left(\gamma_{x} / \gamma_{z}\right.$, see inset) and the decreasing spin lifetime for $B=0$ at temperatures above $120 \mathrm{~K}$ will be discussed in the following. At low temperatures the spin dephasing is governed by the Bir-Aronov-Pikus (BAP) mechanism where the randomly oriented hole spins act via spin interaction with electron spins like a fluctuating effective magnetic field in all directions 15]. The main contribution of the effective magnetic field arises from the 1s exciton where the electron hole overlap is at maximum 16. In a quantum well the $z$ component of the effective magnetic field is dominant, which explains the anisotropy $\gamma_{x} / \gamma_{z}>1$ at low temperatures [17. Higher temperatures break the strong spatial correlation of electrons and holes which leads to the observed increase in spin lifetime up to $80 \mathrm{~K}$. Another clear hint for the dominance of dephasing via the BAP mechanism at low temperatures is found in the time resolved PL transients, where we observed a retarded sub-exponential decay of the degree of spin polarization. Here the BAP mechanism decreases in time due to loss of holes by optical recombination (not shown) [18]. 
For $B=0.6 \mathrm{~T}$ and $T>80 \mathrm{~K}$, the $\mathrm{DP}$ mechanism becomes stronger than the BAP mechanism and leads to the expected reduced spin lifetime $\left[\left(\gamma_{x}+\gamma_{z}\right) / 2\right]^{-1}$. In contrast, the decrease of spin lifetime $\gamma_{z}^{-1}$ for $B=0$ and $T>120 \mathrm{~K}$ is very surprising, since all subbands strictly exhibit no in plane component of the effective magnetic field. Occupation of higher subbands (about 13\% for the first excited subband at $250 \mathrm{~K}$ compared to the lowest band) at elevated temperatures should also not lead to spin dephasing. This can be seen by transforming eq. (1) into the (110) coordinate system

$$
H_{\text {spin }}=\frac{1}{2} \hbar\left[\sigma_{x} \Omega_{x}+\sigma_{y} \Omega_{y}+\sigma_{z} \Omega_{z}\right]
$$

where $\left(\Omega_{x}, \Omega_{y}, \Omega_{z}\right)=\Gamma / \hbar\left(-k_{x}^{2} k_{z}-2 k_{y}^{2} k_{z}+\right.$ $\left.k_{z}^{3}, 4 k_{x} k_{y} k_{z}, k_{x}^{3}-2 k_{x} k_{y}^{2}-k_{x} k_{z}^{2}\right)$. Since $\left\langle k_{z}\right\rangle=\left\langle k_{z}^{3}\right\rangle=0$ for all subbands, all in plane magnetic field components $\Omega_{x}$ and $\Omega_{y}$ vanish. A simple application of the DP dephasing formula $\gamma_{z}=\left\langle\Omega_{x}^{2}+\Omega_{y}^{2}\right\rangle \tau_{p}^{*}=0$ yields no spin dephasing [ $[6]$.

In the following we resolve the puzzle by noting that scattering of electrons between subbands along with spin orbit coupling $H_{\text {spin }}$ constitutes a new spin relaxation mechanism. Existing theories treat spin dephasing only within the lowest subband [19, 20, 21, 22]. Here, we sketch the principle idea for intersubband spin relaxation (ISR) and give a first estimate of its effectiveness. In the case $H_{\text {spin }}=0$ a simultaneous intersubband and spin flip transition like $|\vec{k}, 0, \uparrow\rangle \longrightarrow\left|\overrightarrow{k^{\prime}}, 1, \downarrow\right\rangle$ is forbidden. However, for $H_{\text {spin }} \neq 0$ the corresponding transition $|\vec{k}, 0, \uparrow\rangle+\epsilon_{1}|\vec{k}, 1, \downarrow\rangle+\ldots \longrightarrow\left|\overrightarrow{k^{\prime}}, 1, \downarrow\right\rangle+\epsilon_{2}\left|\overrightarrow{k^{\prime}}, 0, \uparrow\right\rangle+\ldots$ is modified according to first order perturbation theory which mixes zero order terms with first order terms possessing opposite spin. Therefore spin flip transitions become allowed with rates that are $\alpha=\left|\epsilon_{1}\right|^{2}+\left|\epsilon_{2}\right|^{2}$ slower than the intersubband transition rate $\tau_{\mathrm{IB}}^{-1}$, i.e. $\tau_{s} \approx \alpha^{-1} \tau_{\text {IB }}$. We obtain the first order coefficient $\epsilon_{1}\left(k_{x}, k_{y}\right)=\left\langle\vec{k}, 0, \uparrow\left|H_{\text {spin }}\right| \vec{k}, 1, \downarrow\right\rangle / E_{\mathrm{ISG}}$ using a spin splitting constant $\Gamma=19.55 \mathrm{eV}^{3}$ [23], an intersubband gap of $E_{\mathrm{ISG}}=31 \mathrm{meV}$ and approximating the $z$ wavefunctions with that of a well with infinite barriers. We find a spin lifetime $\tau_{s}=\langle\alpha\rangle^{-1} \tau_{\mathrm{IB}}=1.7 \mathrm{~ns}$, for an intersubband scattering rate $\tau_{\mathrm{IB}}=250 \mathrm{fs}$ and a distribution of electrons at $300 \mathrm{~K}$ with averaged $\alpha$ (effective mass $\left.m^{*}=0.0665 m_{0}\right)$. The estimate is in good agreement with our measurements. ISR depends strongly on the occupation of higher subbands and will be much weaker in narrow quantum wells where intersubband splitting is large. This is in accord with recent measurements on an $8.3 \mathrm{~nm}$ thick n-doped GaAs QW that showed spin lifetimes of up to $20 \mathrm{~ns}$ at room temperature 24]. We note that our new dephasing mechanism may also explain a result from Ohno where he found an increased spin lifetime in higher mobility (110) samples at room temperature $\left(\tau_{s} \propto \tau_{p}^{*}, \tau_{\text {IB }}\right.$ respectively), which he could not explain with the usual DP result $\left(\tau_{s} \propto\left(\tau_{p}^{*}\right)^{-1}\right)[\underline{8}]$.
Decreasing spin lifetimes for elevated temperatures have also been reported in (110) grown samples with external electric field. This field induces a Rashba term $\left(\Omega_{x}, \Omega_{y} \neq 0\right)$ which leads via the DP mechanism to spin relaxation [25]. In our sample, the Rashba terms should be very weak or absent since asymmetry of the QW or a built in electric field in growth direction is suppressed by symmetric growth and symmetric doping. We moreover found no experimental evidence for the presence of a Rashba contribution which should reveal itself in spin lifetimes which strongly depend on the orientation of a tilted external magnetic field $\left(B_{x} \cos (\theta), B_{y} \sin (\theta), B_{z}\right)$ if $B_{z} \neq 0$ [26]. And even if some Rashba related spin relaxation was present in our sample, the above estimate shows that the novel ISR mechanism contributes substantially.

In the last paragraph we argue and experimentally verify that anisotropic spin dephasing modifies the usual linear relation $\omega=g \mu_{\mathrm{B}} B / \hbar$ between magnetic field $B$ and measured frequency $\omega$ of spin oscillations, where $\mu_{\mathrm{B}}$ is Bohr's magneton and $g$ is the constant Landé g-factor. The time dependent degrees of spin polarization $s_{x}$ and $s_{z}$ for spins which precess around a magnetic field oriented along $y$ are given by

$$
\frac{\partial}{\partial t}\left(\begin{array}{c}
s_{x} \\
s_{z}
\end{array}\right)=-\left(\begin{array}{cc}
\gamma_{x} & -\omega \\
\omega & \gamma_{z}
\end{array}\right)\left(\begin{array}{c}
s_{x} \\
s_{z}
\end{array}\right)
$$

where $\gamma_{x}$ and $\gamma_{z}$ are the relevant components of the spin dephasing tensor. The solution for $s_{z}(t)$ which is measured in our experiments is

$$
s_{z}(t)=s_{0} \frac{e^{-\left(\gamma_{x}+\gamma_{z}\right) t / 2}}{\cos \varphi} \cos \left(\omega_{\mathrm{a}} t-\varphi\right),
$$

where $\tan \varphi=\frac{\gamma_{x}-\gamma_{z}}{2 \omega_{\mathrm{a}}}, \omega_{\mathrm{a}}=\sqrt{\omega^{2}-\left(\gamma_{x}-\gamma_{z}\right)^{2} / 4}$, and $s_{z}(0)=s_{0}$ is the initial degree of spin polarization. The effective spin oscillation frequency $\omega_{\mathrm{a}}$ is directly affected by the dephasing anisotropy $\gamma_{x}-\gamma_{z}$. The slowing down of the spin oscillations for anisotropic spin dephasing has a pictorial explanation. The magnetic field forces the initial spin orientation into the $x$ direction. The fast $x$ dephasing reduces the $x$ component, whereas the slow $z$-dephasing leaves the $z$-component nearly unchanged. Reducing the $x$ component drives the spin orientation back towards the $z$ axis effectively reducing the precession frequency. The result is a delayed rotation of the spin into the plane of the QW giving rise to a phase shift $\varphi$ of the spin oscillations. After crossing the plane of the QW, the spin is accelerated by the same effect towards the $-z$ direction. On average the spin precession frequency is reduced. Figure 3 (a) depicts the measured modified oscillation frequency $\omega_{\mathrm{a}}$ for magnetic fields between 0.075 and $1 \mathrm{~T}$ along with a fit of $\omega_{\mathrm{a}}(B)$. The value of $\gamma_{x}-\gamma_{z}=1 / 380 \mathrm{ps}^{-1}$ obtained from the fit compares very well with the expected value of $1 / 410 \mathrm{ps}^{-1}$ extracted from the data in Fig. 22 The most significant influence 


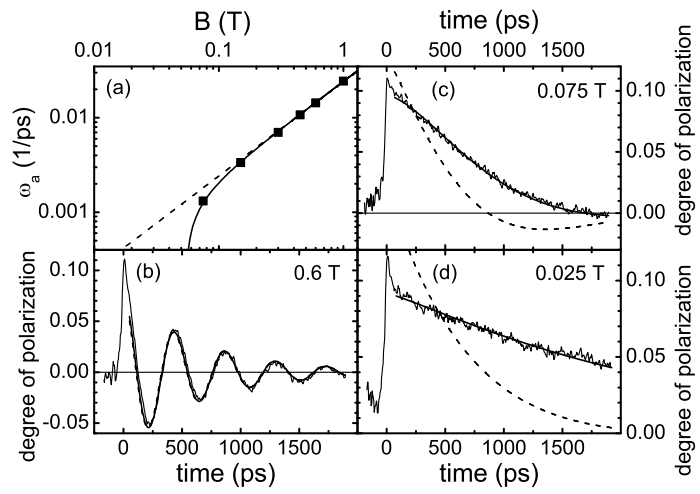

FIG. 3: (a) Measured spin oscillation frequency $\omega_{\mathrm{a}}$ vs. magnetic field (closed squares) at $T=200 \mathrm{~K}$. The fitting curve includes effects of anisotropic spin dephasing (black line). The dashed line depicts for comparison the spin oscillation frequency for isotropic spin dephasing. (b)-(d) Transients of the degree of polarization of time resolved PL for magnetic fields of $0.6,0.075$, and $0.025 \mathrm{~T}$. The black lines are fits including effects of anisotropic spin dephasing. The dashed lines show fits assuming no anisotropy.

of anisotropic spin dephasing is found at magnetic fields below 0.1 T. Figure 3(b) - 3(d) shows the measured time resolved degree of spin polarization along with the theoretical curves including anisotropy [Eqn. (5)] and without anisotropy, i.e. $s_{z}(t)=s_{0} e^{-\left(\gamma_{x}+\gamma_{z}\right) t / 2} \cos (\omega t)$, where we used the initial degree of polarization $s_{0}$ as the only fit parameter. The values for the parameters used for calculating the transients were obtained as follows: $\gamma_{z}$ by fitting the transient at $B=0$ and $g$ and $\gamma_{x}$ by fitting the transients at elevated magnetic fields $(0.075$ to $1 \mathrm{~T})$, assuming validity of anisotropic spin dephasing. While the transient at $B=0.6 \mathrm{~T}$ is matched by both theoretical curves [Fig. 3 (b)], the transients for $B=0.075 \mathrm{~T}$ and $B=0.025 \mathrm{~T}$ can only be explained by including anisotropic spin dephasing. We note that for the case of $|\omega|<\left|\gamma_{x}-\gamma_{z}\right| / 2$, i.e. $B<0.05 \mathrm{~T}$, spin oscillations are completely suppressed.

In conclusion we investigated anisotropic spin dephasing in (110) QWs from $6 \mathrm{~K}$ up to room temperature. Spin memory at room temperature lasts for times as long as nanoseconds only if the spin is parallel or antiparallel to the growth direction. Spin coherence in the plane of the QW is rapidly lost by the DP mechanism. These results have an immediate impact on design considerations for spintronic devices. We further found that intersubband scattering constitutes a new spin dephasing mechanism that is necessary to explain reduced spin lifetimes at elevated temperatures for electron spins along (110). We expect that this new mechanism will stimulate the development of a more general theory of spin dephasing in QWs including the influence of higher subbands. Finally we demonstrated the modification and total suppression of spin oscillations via anisotropic spin dephasing.
We gratefully acknowledge most helpful discussions with R. Winkler and financial support by BMBF and DFG (German Science Foundation).

[1] S. Wolf et al., Science 294, 1488 (2001).

[2] A. Imamoglu et al., Phys. Rev. Lett. 83, 4204 (1999).

[3] J. Rudolph et al., Appl. Phys. Lett. 82, 4516 (2003).

[4] Through all the paper the terms spin decoherence, dephasing, and relaxation are used as synonyms.

[5] M. Dyakonov and V. Perel, Sov. Phys. Solid State 13, 3023 (1972).

[6] F. Meier and B. P. Zakharchenya, Optical orientation (Elsevier Science Publ., Amsterdam, 1984).

[7] J. Kainz, U. Rössler, and R. Winkler, Phys. Rev. B 68, 075322 (2003).

[8] Y. Ohno et al., Phys. Rev. Lett. 83, 4196 (1999).

[9] M. I. Dyakonov and V. Y. Kachorovskii, Sov. Phys. Semicond. 20, 110 (1986).

[10] T. Hassenkam et al., Phys. Rev. B 55, 9298 (1997).

[11] The width of the luminescence peak increase with temperature from 3.5 to $12 \mathrm{~nm}$. For temperatures above $100 \mathrm{~K}$ we used the low energy side of the PL spectrum to determine spin polarization (peak to lower energetic half of the hh transition). A possible contribution of the broadened light hole transition above $50 \mathrm{~K}$ can lower the degree of PL polarization but does not obscure spin relaxation data, because the ratio of hh and lh emission exhibits no transient change. Holes are at elevated temperatures almost instantly in thermal equilibrium.

[12] A. P. Heberle, W. W. Rühle, and K. Ploog, Phys. Rev. Lett. 72, 3887 (1994).

[13] Previously only a very weak anisotropic spindephasing had been reported in strained bulk semiconductors by Dyakonov in 1982. See G. E. Pikus and A. N. Titkov p. 125 in 6] and references.

[14] M. W. Wu and M. Kuwata-Gonokami, Solid State Commun. 121, 509 (2002).

[15] G. L. Bir, A. G. Aronov, and G. E. Pikus, Sov. Phys. JETP 42, 705 (1976).

[16] H. Fu, L.-W. Wang, and A. Zunger, Phys. Rev. B 59, 5568 (1999).

[17] E. Blackwood et al., Phys. Rev. B 50, 14246 (1994).

[18] The spin dephasing times for $B=0$ in Fig. 2 at low temperatures are deduced from the best exponential fit in the time interval between 0 and $1.8 \mathrm{~ns}$.

[19] M. W. Wu and H. Metiu, Phys. Rev. B 61, 2945 (2000).

[20] W. H. Lau, J. T. Olesberg, and M. E. Flatté, Phys. Rev. B 64, 161301 (2001).

[21] M. M. Glazov and E. L. Ivchenko, JETP Letters 75, 403 (2002).

[22] V. I. Puller, L. G. Mourokh, N. J. M. Horing, and A. Y. Smirnov, Phys. Rev. B 67, 155309 (2003).

[23] R. Winkler, Spin-Orbit Coupling Effects in Two-Dimensional Electron and Hole Systems (Springer, Berlin, 2003).

[24] T. Adachi, Y. Ohno, F. Matsukura, and H. Ohno, Physica $\mathrm{E} \mathrm{10,} 36$ (2001).

[25] O. Z. Karimov et al., Phys. Rev. Lett. 91, 246601 (2003).

[26] D. Hägele et al., unpublished. 